\title{
A Review on Macroscopic Pedestrian Flow Modelling
}

\author{
Anna Kormanová ${ }^{1}$ \\ ${ }^{1}$ Faculty of Management Science and Informatics, University of Žilina \\ Univerzitná 8215/1, 01026 Žilina, Slovak Republic \\ Anna.Kormanova@fri.uniza.sk
}

\begin{abstract}
This paper reviews several various approaches to macroscopic pedestrian modelling. It describes hydrodynamic models based on similarity of pedestrian flow with fluids and gases; first-order flow models that use fundamental diagrams and conservation equation; and a model similar to LWR vehicular traffic model, which allows non-classical shocks. At the end of the paper there is stated a comparison of described models, intended to find appropriate macroscopic model to eventually be a part of a hybrid model. The future work of the author is outlined.
\end{abstract}

Keywords: Flow modelling, Macroscopic model, Pedestrian dynamics 


\section{INTRODUCTION}

Pedestrian simulation has wide usage in praxis these days. People tend to group in relatively small places. Very crowded places can be transportation terminals - airports, railway stations, subways, but also theatres, stadiums, etc. There comes a need to solve building trafficability and a layout of serving points, information tables, emergency exits, etc. It can not only help to save financial resources, but also human lives.

However, the choice of modelling method can be difficult. We have to consider especially purpose of the simulation and needed degree of granularity. Granularity in this context means the degree of the modelling detail. From this point of view models are divided into microscopic, mesoscopic and macroscopic. Each has its pros and cons. Microscopic models model pedestrians in great detail, distinguish individuals and their interactions. Macroscopic models on the other hand model pedestrian flow as a whole and can be used in situations, where human interaction is not closely studied. Mesoscopic modeling usually consist of simplification of dynamics with less data demand. It combines microscopic aspects (they can deal with individual pedestrians) and macroscopic aspects, such as flow dynamics. Therefore very interesting and also effective alternative seems to be the creation of a hybrid simulation model that would allow to flexibly define and modify the granularity for different areas of modeled scene.

The simulation tool PedSim for pedestrian movement [11][12] is being developed at the Department of Transportation Networks at Faculty of Management Science and Informatics for several years. The goal is to develop a complex, generic, agent based 3D tool for modeling pedestrians' movement. The utilization of the model can be found in evacuation and design evaluation scenarios of airports, transportation terminals etc. This tool utilizes microscopic and mesoscopic models of pedestrian movement so far. Our goal is to add macroscopic model as well, and create a hybrid simulation model. Macroscopic modelling will be used in areas, which are not statistically important, or where human interaction observation is not necessary. Microscopic and macroscopic display of PedSim is depicted on figure 1.
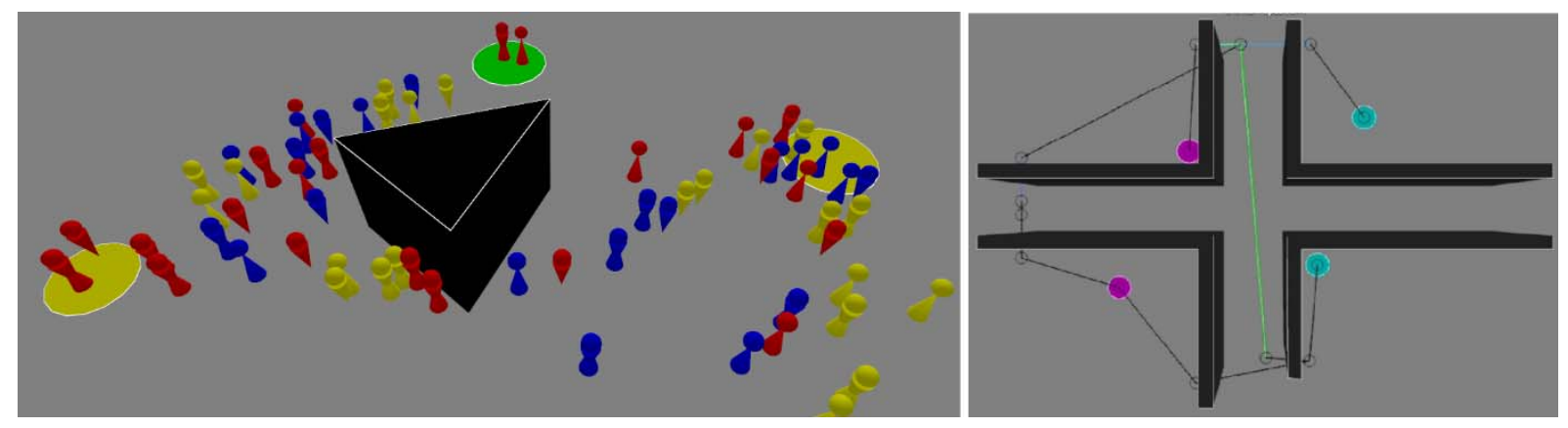

Fig. 1. Left - microscopic movement in PedSim. Right - macroscopic movement in PedSim. Flow is moving through edges of macroscopic graph. No individuals are distinguished, we can only see value of crowd density in the edge determined by corresponding color (color palette from blue to red, red is the highest density).

This paper provides a review of several macroscopic flow models. Their advantage is, that they do not require as much computational power as microscopic methods. At the end of the article there is a 
comparison of described models. This provides an information, which one of models is the most suitable for our purpose of creating a hybrid simulation model for PedSim simulation tool.

\section{HYDRODYNAMIC MODELS}

Hydrodynamic models [8] use analogy with fluids or gases to describe density and speed changes in time. These models are based on a fact, that pedestrian flows and their mesoscopic and macroscopic characteristics are driven by physical laws similar to those valid for dynamics of compressed fluid or gases.

This analogy was studied by number of scientists, who derived some hydrodynamic models out of it. Some of these models, similar to physical ones, are called gas-kinetic models (Boltzmann-like approach, is used mostly for traffic modelling). They describe pedestrian dynamics using distribution function of speed. Other, macroscopic models, use Navier-Stokes type of equations or equations of pedestrian conservation.

Compared to traffic hydrodynamic models, the number of pedestrian models is low, since the complexity of human behavior is much larger than vehicle one.

\subsection{HELBING'S FLUID-DYNAMIC MODEL}

Dirk Helbing [6] presented his fluid-dynamic model for the collective movement of pedestrians in 1992. It is based on Boltzmann-like gas-kinetic model. The first one to apply this kind of model on pedestrian crowds was Henderson [7]. However, he assumed a conservation of momentum and energy, what was quite unrealistic.

Pedestrian here belongs into type $\mu$ of motion, if he wants to walk approximately with the velocity $\vec{v}^{0}$. Density $\hat{\rho}_{\mu}\left(\vec{x}, \vec{v}_{\mu}, \vec{v}_{\mu}^{0}, t\right)$ describes a number $N_{\mu}$ of pedestrians of type $\mu$, who are present within an area $A=A(\vec{x})$ around a place $\vec{x}$, with intended velocity of approximately $\vec{v}_{\mu}^{0}$, while the actual velocity is approximately $\vec{v}_{\mu}$. It is defined as:

$$
\hat{\rho}_{\mu}\left(\vec{x}, \vec{v}_{\mu}, \vec{v}_{\mu}^{0}, t\right) \equiv \hat{\rho}_{\mu}\left(\vec{x}, \vec{u}_{\mu}, t\right):=\frac{N_{\mu}\left(U(\vec{x}) \times \mathcal{V}\left(\vec{u}_{\mu}\right), t\right)}{A \cdot V}
$$

$U(\vec{x})$ is a neighborhood around place $\vec{x}$, which represents all accessible (public) places $\vec{x}$.

$$
\mathcal{U}(\vec{x}):=\left\{\vec{x}^{*} \in \mathcal{M}:\left\|\vec{x}^{*}-\vec{x}\right\| \leq r\right\}
$$

Similarly, $\mathcal{V}\left(\vec{u}_{\mu}\right)$ is a neighborhood of $\left(\vec{u}_{\mu}\right)=\left(\vec{u}_{\mu}, \vec{v}_{\mu}^{0}\right)$, with a volume $V=V\left(\vec{u}_{\mu}\right)$.

Next the continuity equations have been established:

$$
\frac{d \hat{\rho}_{\mu}}{d t} \equiv \frac{\partial \hat{\rho}_{\mu}}{\partial t}+\nabla_{\vec{x}}\left(\hat{\rho}_{\mu} \vec{v}_{\mu}\right)+\nabla_{\vec{v}_{\mu}}\left(\hat{\rho}_{\mu} \frac{\vec{f}_{\mu}}{m_{\mu}}\right)+\nabla_{\vec{v}_{\mu}^{0}}\left(\hat{\rho}_{\mu} \vec{v}_{\mu}^{0}\right):=\frac{\hat{\rho}_{\mu}^{0}-\hat{\rho}_{\mu}}{\tau_{\mu}}+\sum_{v} \hat{S}_{\mu v}+\sum_{v} \hat{C}_{\mu v}+\vec{q}_{\mu} .
$$


These can be interpreted as gas-kinetic equations. $m_{\mu}$ denotes the average mass of type $\mu$ pedestrians. The force $\vec{f}_{\mu}=m_{\mu} \vec{v}_{\mu}$ can often be neglected.

The change of density $\hat{\rho}_{\mu}$ over time is influenced by four facts:

- The tendency of pedestrians to reach their intended velocity $\vec{v}_{\mu}^{0}$.

- Interactions among pedestrians.

- Changes of pedestrian types when turning right or left or when at a crossing.

- The density gain or loss per time unit.

By multiplication of (3) by $\psi_{\mu}\left(\vec{v}_{\mu}\right)=m_{\mu}, m_{\mu} \vec{v}_{\mu}$ or $m_{\mu} v_{\mu, i}^{2} / 2$, and integration over $\vec{u}_{\mu}$, we obtain following equations:

$$
\frac{\partial\left\langle\varrho_{\mu}\right\rangle}{\partial t}=-\frac{\partial}{\partial x_{\mu, \alpha}}\left(\left\langle\varrho_{\mu}\right\rangle\left\langle v_{\mu, \alpha}\right\rangle\right)+Q_{\mu}(1)+\sum_{v}\left[\frac{m_{\mu}}{m_{v}}\left\langle\varrho_{\mu}\right\rangle \chi_{\mu}^{v \mu}(1)-\left\langle\varrho_{\mu}\right\rangle \chi_{\mu}^{\mu v}(1)\right]
$$

for the mass density,

$$
\begin{aligned}
\frac{\partial\left\langle\varrho_{\mu}\right\rangle\left\langle v_{\mu, \beta}\right\rangle}{\partial t}=- & \frac{\partial}{\partial x_{\mu, \alpha}}\left(\left\langle\varrho_{\mu}\right\rangle\left\langle v_{\mu, \alpha}\right\rangle\left\langle v_{\mu, \beta}\right\rangle+\left\langle\rho_{\mu, \alpha \beta}\right\rangle\right)+\left\langle\varrho_{\mu}\right\rangle \frac{\left\langle f_{\mu, \beta}\right\rangle}{m_{\mu}}+Q_{\mu}\left(v_{\mu, \beta}\right) \\
& +\left\langle\varrho_{\mu}\right\rangle \frac{1}{\tau_{\mu}}\left(\left\langle v_{\mu, \beta}^{0}\right\rangle-\left\langle v_{\mu, \beta}\right\rangle\right) \\
& +\sum_{v}\left\langle\varrho_{\mu}\right\rangle\left\langle\varrho_{v}\right\rangle \frac{1}{m_{v}}\left[\chi_{\mu v}\left(v_{\mu, \beta}^{*}\right)-\chi_{\mu v}\left(v_{\mu, \beta}\right)\right] \\
& +\sum_{v}\left[\frac{m_{\mu}}{m_{v}}\left\langle\varrho_{\mu}\right\rangle \chi_{\mu}^{v \mu}\left(v_{v, \beta}\right)-\left\langle\varrho_{\mu}\right\rangle \chi_{\mu}^{\mu v}\left(v_{\mu, \beta}\right)\right]
\end{aligned}
$$

for the momentum density, and

$$
\begin{aligned}
\frac{\partial\left\langle\epsilon_{\mu, i}\right\rangle}{\partial t}=-\frac{\partial}{\partial x_{\mu, \alpha}} & \left(\left\langle v_{\mu, \alpha}\right\rangle\left\langle\epsilon_{\mu, i}\right\rangle+\rho_{\mu, \alpha i}\left\langle v_{\mu, i}\right\rangle+j_{\mu, \alpha, i}\right)+\left\langle\varrho_{\mu}\right\rangle\left\langle v_{\mu, i}\right\rangle \frac{f_{\mu, i}}{m_{\mu}}+Q_{\mu}\left(\frac{v_{\mu, i}^{2}}{2}\right) \\
& +\left\langle\varrho_{\mu}\right\rangle \frac{1}{\tau_{\mu}}\left(\left\langle v_{\mu, i}^{0}\right\rangle^{2}-\left\langle v_{\mu, i}\right\rangle^{2}\right)+\left\langle\varrho_{\mu}\right\rangle \frac{1}{\tau_{\mu}}\left(\left\langle\left(\delta v_{\mu, i}^{0}\right)^{2}\right\rangle-\left\langle\left(\delta v_{\mu, i}\right)^{2}\right\rangle\right) \\
& +\sum_{v}\left\langle\varrho_{\mu}\right\rangle\left\langle\varrho_{v}\right\rangle \frac{1}{m_{v}}\left[\chi_{\mu v}\left(\frac{v_{\mu, i}^{*}}{2}\right)-\chi_{\mu v}\left(\frac{v_{\mu, i}^{2}}{2}\right)\right] \\
& +\sum_{v}\left[\frac{m_{\mu}}{m_{v}}\left\langle\varrho_{\mu}\right\rangle \chi_{\mu}^{v \mu}\left(\frac{v_{v, i}^{2}}{2}\right)-\left\langle\varrho_{\mu}\right\rangle \chi_{\mu}^{\mu v}\left(\frac{v_{\mu, i}^{2}}{2}\right)\right]
\end{aligned}
$$

for the energy density. Here, the density of pedestrians of type $\mu$ is $\left\langle\rho_{\mu}\right\rangle$, their velocity $\left\langle v_{\mu}\right\rangle$, and the variance of their velocity components $v_{\mu, i}$ (at place $\vec{x}$ and time $t$ ) is $\left\langle\left(\delta v_{\mu, i}\right)^{2}\right\rangle .\left\langle\varrho_{\mu}\right\rangle:=m_{\mu}\left\langle\rho_{\mu}\right\rangle$ stands for the mass density, the mean momentum density $\left\langle\rho_{\mu}\right\rangle\left\langle m_{\mu} \vec{v}_{\mu}\right\rangle=\left\langle\varrho_{\mu}\right\rangle\left\langle\vec{v}_{\mu}\right\rangle$, and the mean energy density $\left\langle\epsilon_{\mu, i}\right\rangle=\left\langle\rho_{\mu}\right\rangle\left\langle\frac{m_{\mu}}{2} v_{\mu, i}^{2}\right\rangle=\left\langle\varrho_{\mu}\right\rangle \frac{v_{\mu, i}^{2}}{2}+\left\langle\varrho_{\mu}\right\rangle\left\langle\frac{\left(\delta v_{\mu, i}\right)^{2}}{2}\right\rangle$.

Equations (4), (5) and (6) represent the known hydrodynamic equations. Within there is also an interaction between pedestrians of type $\mu$ and $v$ included. Detailed explanation of equations is described in [6]. 


\subsection{HUGHES'S MODEL}

Roger L. Hughes [9][10] proposed a model resulting from three hypotheses:

Hypothesis 1. The speed at which pedestrians walk is determined solely by the density of surrounding pedestrians, the behavioral characteristics of the pedestrians, and the ground on which they walk.

The velocity components $(u, v)$ of a pedestrian are given by

$$
u=f(\rho) \hat{\phi}_{x}, \quad v=f(\rho) \hat{\phi}_{y}
$$

where $\hat{\phi}_{x}$ and $\hat{\phi}_{y}$ are the direction cosines of the motion and $f(\rho)$ is a speed of pedestrian as a function of density.

Hypothesis 2. Pedestrians have a common sense of the task (called potential) that they face to reach their common destination, such that any two individuals at different locations having the same potential would see no advantage to exchanging places.

Thus the direction of a motion of the pedestrian is perpendicular to his potential, i.e. the direction cosines are

$$
\hat{\phi}_{x}=\frac{-(\partial \phi / \partial x)}{\sqrt{(\partial \phi / \partial x)^{2}+(\partial \phi / \partial y)^{2}}} \quad \hat{\phi}_{y}=\frac{-(\partial \phi / \partial y)}{\sqrt{(\partial \phi / \partial x)^{2}+(\partial \phi / \partial y)^{2}}}
$$

where $\phi$ is potential. This hypothesis is applicable for pedestrian flows, because they can visually access the situation.

Hypothesis 3. Pedestrians seek to minimize their estimated travel time but temper this behavior to avoid extreme densities. This tempering is assumed to be separable, such that pedestrians minimize the product of their travel time as a function of density.

The distance between pedestrians' potentials must be proportional to pedestrian speed, thus

$$
\frac{1}{\sqrt{(\partial \phi / \partial x)^{2}+(\partial \phi / \partial y)^{2}}}=\sqrt{u^{2}+v^{2}}
$$

with potential scaled appropriately.

Equations (7)-(9) with the usual continuity equation are combined into equations for pedestrian flow

$$
-\frac{\partial \rho}{\partial t}+\frac{\partial}{\partial x}\left(\rho g(\rho) f^{2}(\rho) \frac{\partial \phi}{\partial x}\right)+\frac{\partial}{\partial y}\left(\rho g(\rho) f^{2}(\rho) \frac{\partial \phi}{\partial y}\right)=0
$$

and

$$
g(\rho) f(\rho)=\frac{1}{\sqrt{(\partial \phi / \partial x)^{2}+(\partial \phi / \partial y)^{2}}}
$$

where factor $g(\rho)$ stands for the tempering behaviour at high densities. 
This formulation can easily be extended to multiple pedestrian types crows. $f(\rho)$ and $g(\rho)$ can be approximated by:

$$
f(\rho)= \begin{cases}A, & \rho \leq \rho_{\text {trans }} \\ A \sqrt{\frac{\rho_{\text {trans }}}{\rho},}, & \rho_{\text {trans }}<\rho \leq \rho_{\text {max }} \\ A \sqrt{\frac{\rho_{\text {trans }} \rho_{\text {crit }}\left(\rho_{\text {max }}-\rho\right)}{\rho^{2}\left(\rho_{\text {max }}-\rho_{\text {crit }}\right)}}, & \rho_{\text {crit }}<\rho \leq \rho_{\text {max }}\end{cases}
$$

and

$$
g(\rho)=\left\{\begin{array}{cl}
1, & \rho \leq \rho_{\text {crit }} \\
\frac{\rho\left(\rho_{\max }-\rho_{\text {crit }}\right)}{\rho_{\text {crit }}\left(\rho_{\max }-\rho\right)}, & \rho_{\text {crit }}<\rho \leq \rho_{\text {max }}
\end{array}\right.
$$

where typically $A=1.4 \mathrm{~ms}^{-1}, \rho_{\text {trans }}=0.8 \mathrm{~ms}^{-1}, \rho_{\text {crit }}=3.0 \mathrm{~ms}^{-1}$, and $\rho_{\text {max }}=5.0 \mathrm{~ms}^{-1}$.

\section{FIRST-ORDER FLOW MODELS}

The first-order pedestrian flow theory [1][4][5] is based on combination of a fundamental diagram and the conservation of pedestrians. Fundamental diagram represents flow-density relation.

\subsection{DAAMEN-HOOGENDOORN-BOVY}

The fundamental diagrams here [4][5] are derived from cumulative flow plots - functions $N(x, t)$ that represent counted number of pedestrians that pass a cross-section $x$ from an arbitrary starting point. The flow $q$ in a cross-section $x$ in time period $t_{1}$ to $t_{2}$ equals:

$$
q\left(x, t_{1} \text { to } t_{2}\right)=\frac{N\left(x, t_{2}\right)-N\left(x, t_{1}\right)}{t_{2}-t_{1}}
$$

The density at point $x$ in time $t$ is derived from fundamental relation between speed $u$, density $k$ and flow $q$ :

$$
k(x, t)=\frac{q(x, t)}{u(x, t)}
$$

In [5] the three-dimensional cell $C$ with dimensions $X \times Y \times T$ is considered. For every trajectory passing the cell, the three quantities are determined:

1. The travel time $T T_{i}-$ duration of pedestrian $i$ in the cell. $0<T T_{i} \leq T$

2. The travelled distance $D_{i}$ in the $x$-direction - the distance pedestrian $i$ walks in $x$-direction while staying in the cell. $0<D_{i} \leq X$

3. The travelled distance $Z_{i}$ in the $y$-direction - the distance pedestrian $i$ walks in $y$-direction while staying in the cell. $0<Z_{i} \leq X$

From these quantities, the following equations are generalized: 


$$
\begin{gathered}
k=\frac{\sum_{i \in C} T T_{i}}{X Y T} \\
q_{x}=\frac{\sum_{i \in C} D_{i}}{X Y T} \text { and } q_{y}=\frac{\sum_{i \in C} Z_{i}}{X Y T}
\end{gathered}
$$

where $k$ is density in generalized form in $P / \mathrm{m}^{2}$ and $q$ is definition of flow in $x$ and $y$ direction (in $P / m s)$. If $T T_{i=} T$, then:

$$
k(x, t)=\frac{\sum_{i \in C} T T_{i}}{X Y T}=\frac{n T}{X Y T}=\frac{n}{X Y}
$$

where $n$ is the number of pedestrians in area $X \times Y$ at time $t$. The same holds for flow definitions.

Pedestrian speeds in $x$ and $y$ directions can easily be determined:

$$
v_{x}=\frac{q_{x}}{k}=\frac{\sum_{i \in C} D_{i}}{\sum_{i \in C} T T_{i}} \text { and } v_{y}=\frac{q_{y}}{k}=\frac{\sum_{i \in C} Z_{i}}{\sum_{i \in C} T T_{i}}
$$

Based on the data of narrow bottleneck experiment, the fundamental graph has been constructed.

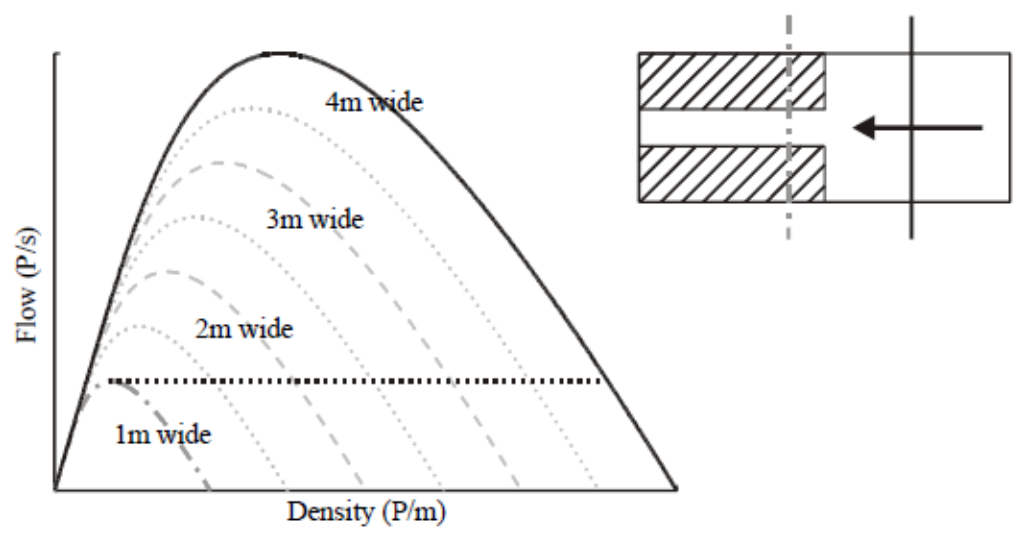

Fig. 2. Fundamental diagram for pedestrian traffic for bottleneck experiment. [5]

\subsection{BRUNO ET. AL.}

Bruno et. al. [1] proposed physical framework based on several features of pedestrian behaviour:

(f1) pedestrians are active agents, i.e. in normal (non-panic) situations, their shared objective is to walk with the maximum velocity towards a target $\mathcal{T}$ (doors, exits, etc.), while avoiding obstacles and crowded zones. They actively determine their walking direction and velocity and are not passively subjected to the laws of inertia; 
(f2) pedestrians are intelligent agents, so their mind evaluates, selects, makes synthesis of its perceptions according to various psychological criteria.

(f3) Pedestrians under normal conditions do not perceive the real world locally in space, but have the ability to see up to a given extent around them. The area required for perception, evaluation and reaction is called sensory region and is notated as $R_{s}$;

(f4) pedestrians react after a reflex time delay $\tau_{1}$ from the perception time, which is needed for reaction to the actual conditions, while a volitional time delay $\tau_{2}$ is required to adopt walking strategies and to scan visual field. $\tau_{1} \ll \tau_{2} \ll T, T$ is the reference macroscopic time scale required to cross the walking area at maximum walking speed $v_{M}$;

(f5) from the previous it comes, that pedestrians in a given position at a given time react to perceived conditions in front of them at a delayed time, i.e. in a non-local way in both space and time.

(f6) Pedestrians are anisotropic agents, they distinguish between ahead and behind. In normal situations they are essentially sensitive to what happens in a visual field focused on their direction of movement;

(f7) walking pedestrians adapt their sensory region's depth and width to their travel purpose and walking speed.

Features f1-f2 are about intelligent behavior, features f3-f5 about non-local behavior, and features f6$\mathrm{f} 7$ about anisotropic behavior.

\subsubsection{PHYSICAL MODELLING FRAMEWORK}

The proposed model is expressed by a mass conservation equation for a control mass $M$ in time $t$ :

$$
\frac{D M}{D t}=0
$$

To describe a system dynamics, the velocity field of the moving mass should be a state variable or be a priori known. First-order models relate the velocity field to the mass itself.

The velocity field $\mathbf{v}$ in the point $\mathbf{x} \epsilon \Omega$ at time $t$ :

$$
\mathbf{v}(\mathbf{x}, t)=v(\mathbf{x}, t) \mathbf{e}_{v}(\mathbf{x}, t)
$$

where $v$ is the magnitude of $\mathbf{v}$ (i.e. the walking speed) and $\mathbf{e}_{v}$ is a unit vector of walking direction. The walking direction is modelled as the superposition of two contributors:

- the desired direction identified by the unit vector $\mathbf{e}_{d}$;

- the interaction direction - the direction, the pedestrian would follow to avoid crowded areas. It is identified by the unit vector $\mathbf{e}_{i}$, which, unlike $\mathbf{e}_{d}$, evolves in time.

The desired direction is a priori determined. The interaction direction is instead obtained through a perception process. The resulting walking direction $\mathbf{e}_{v}$ is:

$$
\mathbf{e}_{v}=\frac{\theta \mathbf{e}_{d}+(1-\theta) \mathbf{e}_{i}}{\left|\theta \mathbf{e}_{d}+(1-\theta) \mathbf{e}_{i}\right|},
$$


Where $\theta \in[0,1]$ is a dimensionless parameter - weight of pedestrian's attitude to prioritize the walking area layout or the crowd conditions.

The walking speed $v$ is determined by:

$$
v=v_{M}\left\{1-\exp \left[-\gamma\left(\frac{1}{\rho_{p}}-\frac{1}{\rho_{M}}\right)\right]\right\}
$$

where $v_{M}$ is the free speed, $\rho_{M}$ is the jam density, $\gamma$ is an exponent, that makes the relation sensitive to different travel purposes, $\rho_{p}=\rho_{p}(\mathbf{x}, t)$ is the perceived density.

The sensory region $R_{S}=R_{S}\left(\mathbf{x}, \mathbf{e}_{d}, \delta, \bar{\alpha}\right)$ is modelled as a portion of ball centered in $\mathbf{x}$ with radius $\delta$ and angular span $2 \bar{\alpha}$. In the $1 \mathrm{D}$ case the definition is simple:

$$
R_{S}(x, \delta)=[x, x+\delta] \subseteq[0, L],
$$

where the interval $\Omega=[0, L]$ is the $1 \mathrm{D}$ domain. It is assumed that the depth $\delta$ depends on the walking speed only, while the width $2 \bar{\alpha}$ is a constant. The radius $\delta$ is expressed as a function of the local delayed walking speed.

$$
\delta(\mathbf{x}, t)=\delta\left(v\left(\mathbf{x}, t-\tau_{1}\right)\right)
$$

The constitutive law linking $\delta$ to the walking speed is as follows:

$$
\delta(v)=\frac{\Delta_{s}}{v_{M}}+\delta_{0}
$$

where $\Delta_{s}$ and $\delta_{0}$ are understood as the macroscopic counterparts of $d\left(v_{M}\right)$ and $\delta_{0}$. It is assumed that $\delta_{0}$ is constant equaling a given fraction of the reference length $L$.

The visual field width $\bar{\alpha}$ varies in range $\left[0,90^{\circ}\right]$. The crowd density and the interaction direction (in $2 \mathrm{D}$ setting) come from an intelligent evaluation process.

\section{COLOMBO-ROSINI MODEL}

A macroscopic model presented by [2][3] can be used to describe real situations, such as crowd evacuating a corridor. It is a one-dimensional model built on a Couchy problem for the nonlinear hyperbolic conservation law

$$
\partial_{t} \rho+\partial_{x} f(\rho)=0, \quad x \in \mathbb{R}, t>0,
$$

which reminds of the Lighthill-Whitham-Richards (LWR) model of vehicular traffic. Furthermore, it is able to reproduce the Braess' paradox for pedestrians and was experimentally confirmed by an empirical study.

When the density grows above the maximum value, it is assumed, that unlike vehicles, pedestrians can still move but feel overcompressed. As described in [2], this allows to define a concept of solution to the above conservation law in which non-classical shocks are admitted. 


\section{COMPARISON OF MACROSCOPIC MODELS}

Described models were compared from several points of view, as stated in table 1 . They are all based on physical laws.

Helbing's model is based on fluid-dynamics. As one of a few it is applicable also on panic situations, what allows us to model evacuations. Different types of pedestrians are distinguished here. This model describes developments of walking lanes, propagation of waves and behavior on a dance floor. It has wide application possibilities. Only problem can arise in places with very low densities. The greatest disadvantage, however, is the fact, that the model has not been validated.

Hugh's model is based on well-defined hypotheses. It describes time-dependent waves and Braess' paradox. This model also has problem with low densities. It has been properly validated, but cannot be used in panic situations.

Daamen's model is based on flow-density diagram. It has been validated only by laboratory experiments. It is applicable on pedestrian traffic, but not on panic situations. No apparent problems have been described.

Bruno et. al. designed model based on physical model and crowd dynamics. It is the only model which recognizes individual pedestrians. It can be used for pedestrian traffic modelling, but does not support panic situations. The diffusive effect is explained.

Colombo-Rosini model is based on LWR model. It described phenomena of shock waves and Braess' paradox. Its advantage is applicability to panic situations and evacuations.

Since our simulation tool PedSim focuses on evacuation and evaluation scenarios mostly at large transportation terminals, a good choice seems to be the Colombo-Rosini model, which allows panic situations. As the only model from described ones, this one is focused on evacuations. Unlike Helbing's model, which also allows panic situations, Colombo-Rosini model has been properly validated. 


\begin{tabular}{|c|c|c|c|c|c|}
\hline & HELBING & HUGH & $\begin{array}{l}\text { DAAMEN } \\
\text { et. al. }\end{array}$ & $\begin{array}{l}\text { BRUNO et. } \\
\text { al. }\end{array}$ & $\begin{array}{l}\text { COLOMBO- } \\
\text { ROSINI }\end{array}$ \\
\hline Model based on & Fluid-dynamics & $\begin{array}{l}\text { Well-defined } \\
\text { observations } \\
\text { (hypotheses) }\end{array}$ & $\begin{array}{l}\text { Flow-density } \\
\text { diagram }\end{array}$ & $\begin{array}{l}\text { Physical } \\
\text { model and } \\
\text { crowd } \\
\text { dynamics }\end{array}$ & $\begin{array}{l}\text { Altered LWR } \\
\text { model }\end{array}$ \\
\hline Possible problems & Low densities & Low densities & $\begin{array}{c}\text { Not } \\
\text { described }\end{array}$ & Not described & $\begin{array}{c}\text { Describes only } \\
\text { one dimensional } \\
\text { movements }\end{array}$ \\
\hline $\begin{array}{c}\text { Applicable to panic } \\
\text { situations }\end{array}$ & Yes & No & No & No & Yes \\
\hline $\begin{array}{l}\text { Recognizes } \\
\text { individuals }\end{array}$ & $\begin{array}{l}\text { Only groups of } \\
\text { different types }\end{array}$ & No & No & Yes & No \\
\hline Value of variables & Physical meaning & $\begin{array}{l}\text { Physical } \\
\text { meaning }\end{array}$ & $\begin{array}{l}\text { Physical } \\
\text { meaning }\end{array}$ & $\begin{array}{l}\text { Physical } \\
\text { meaning }\end{array}$ & $\begin{array}{l}\text { Physical } \\
\text { meaning }\end{array}$ \\
\hline $\begin{array}{l}\text { Parameters } \\
\text { calibration }\end{array}$ & By inspection & Typical values & By inspection & By inspection & By inspection \\
\hline Validated & No & Yes & $\begin{array}{l}\text { Laboratory } \\
\text { experiments }\end{array}$ & Yes & Yes \\
\hline $\begin{array}{l}\text { Phenomena } \\
\text { explained }\end{array}$ & $\begin{array}{c}\text { Development of } \\
\text { walking lanes, } \\
\text { propagation of waves, } \\
\text { behavior on a dance } \\
\text { floor }\end{array}$ & $\begin{array}{c}\text { Time-dependent } \\
\text { waves, Braess' } \\
\text { paradox }\end{array}$ & $\begin{array}{c}\text { None } \\
\text { described }\end{array}$ & $\begin{array}{l}\text { Diffusive } \\
\text { effect }\end{array}$ & $\begin{array}{c}\text { Braess' paradox, } \\
\text { shock waves }\end{array}$ \\
\hline Possible applications & $\begin{array}{l}\text { Town- and traffic- } \\
\text { planning }\end{array}$ & Crowd motion & $\begin{array}{l}\text { Pedestrian } \\
\text { traffic }\end{array}$ & $\begin{array}{l}\text { Pedestrian } \\
\text { traffic }\end{array}$ & Evacuation \\
\hline
\end{tabular}

Table 1. Comparison of macroscopic models.

\section{CONCLUSION}

Macroscopic models save computation time significantly. They are convenient to use when pedestrian to pedestrian interaction is not needed to be closely studied.

At the Department of Transportation Networks of Faculty of Management Science and Informatics of University of Žilina we are developing a simulation tool PedSim [11, 12] for simulating pedestrian movement. Our goal is to create hybrid model - combination of microscopic and macroscopic model. Microscopic model will be used in complicated areas or areas of interest, and macroscopic in the rest. From the comparison of several flow models we can see, that the most suitable one is Colombo-Rosini model, which is applicable to panic situations and evacuations.

The future work will be focused on implementation and calibration of selected macroscopic model into PedSim simulation tool. Finally, the transitions and synchronization between microscopic and macroscopic models will be solved and hybrid model will be created. 


\section{REFERENCES}

[1] BRUNO, L., TOSIN, A., TRICERRI, P., VENUTI, F. Non-local first-order modelling of crowd dynamics: A multidimensional framework with applications, Applied Mathematical Modelling. vol. 35, no. 1, pp. 426-445, 2011. Available from: http://arxiv.org/pdf/1003.3891v2.pdf

[2] COLOMBO, R. M., GOATIN, P., ROSINI, M. D. A macroscopic model for pedestrian flows in panic situations, in GAKUTO International Series Mathematical Sciences and Applications. 2010, pp. 255-272. Available from: http://hal.archivesouvertes.fr/docs/00/53/48/88/PDF/43-rosini.pdf

[3] COLOMBO, R. M., ROSINI, M. D. Pedestrian flows and non-classical shocks. Mathematical Methods in the Applied Sciences, vol. 28, no. 13, pp. 1553-1567, 2005. Available from: http://citeseerx.ist.psu.edu/viewdoc/download?doi=10.1.1.105.6594\&rep=rep1\&type=pdf

[4] DAAMEN, W. Modelling Passenger Flows in Public Transport Facilities, dissertation thesis, 2004. Available from: http://repository.tudelft.nl/assets/uuid:e65fb66c-1e55-4e63-8c495199d40f60e1/trail_daamen_20040909.pdf

[5] DAAMEN, W., HOOGENDOORN, S. P., BOVY, P. H. L. First-order pedestrian traffic flow theory, Transportation Research Record: Journal of the Transportation Research Board, vol. 1934, no. 1, pp. 43-52, 2005. Available from: http://www.researchgate.net/publication/228531222_Firstorder pedestrian traffic flow theory/file/79e41509d5b3c90d97.pdf

[6] HELBING, D. A Fluid-Dynamic Model for the Movement of Pedestrians, in Complex Systems 6, 1992, pp. 391-415. Available from: http://www.complex-systems.com/pdf/06-5-1.pdf

[7] HENDERSON, L. F. On the fluid mechanics of human crowd motion, Transportation research, vol. 8, pp. 509-515, 1974.

[8] HOOGENDOORN, S. P., BOVY, P. H. L. Normative pedestrian behaviour theory and modeling, in Proceedings of the 15th International Symposium on Transportation and Traffic Theory, 2002.

[9] HUGHES, R. L. The Flow of Human Crowds, Annual review of fluid mechanics, vol. 35, pp. 169-182, $2003 . \quad$ Available from: http://www.computingscience.nl/docs/vakken/mcrs/papers/15.pdf

[10] HUGHES, R. L. The flow of large crowds of pedestrians, Mathematics and Computers in Simulation, vol. 53, pp. 367-370, 2000. Available from: http://www.sciencedirect.com/science/article/pii/S0378475400002287\#

[11] KORMANOVÁ, A. Combining Social Forces and Cellular Automata Models in Pedestrians' Movement Simulation, Journal of Information, Control and Management Systems, vol. 10, no. 1, pp. 61-70, 2012.

[12] VARGA, M. Application of the ABAsim architecture in the pedestrian movement modeling tool, Journal of Information, Control and Management Systems, vol. 10, no. 1, pp. 125-134, 2012. 